\title{
AN EVALUATION OF COLLEGE PERSONNEL WORK IN TERMS OF CURRENT RESEARCH ON INTERPERSONAL RELATIONSHIPS
}

\author{
JOHN H. ROHRER \\ University of Oklahoma ${ }^{1}$ \\ Introduction
}

Is planning this paper it was my intention to develop it along three lines: (I) To present an analysis of the societal functions which higher education served, and the role of the college personnel worker in the performance of those functions. (2) To point out some little-used but valuable methodological research techniques which should aid the college personnel worker to obtain meaningful answers concerning the problems which confront him in the field of teaching students skills in interpersonal relationships. (3) To review some representative research studies in the field of the social sciences that are pertinent to the problems of college personnel work. When I had completed the rough draft, I had just about enough material to last four times the length of time alotted me. In cutting it was necessary to present only the major conclusions of the first section without any documentation of the ways in which those conclusions were reached and, in addition, to make bare mention of the valuable research methodologies which are little used in personnel work. For those who may be interested, I plan to publish the first section in its detailed form. In the published report of this paper will be found references to a detailed consideration of the methodological studies mentioned.

\section{The Societal Responsibilities of Higher Education and the Role of the College Personnel Worker in Meeting Those Responsibilities}

An evaluation of the societal functions to be served by institutions of higher education, as revealed by an analysis of social

${ }^{1}$ On leave for the $1948-49$ school year and working at the Institute of Human Relations, Yale University, under a Social Science Research Council Fellowship award. 
structure, role behaviors and adjustment difficulties unique to the population class, "college graduates," leads to the following five conclusions:

I. Institutions of higher education have as their responsibility the development of those essential personal and interpersonal skills and knowledges which will enable the student to successfully perform the role activities of a particular social status for which the institution purports to train him. Any failure to be continuously aware of this social frame of reference will lead to a neglect of a major area of training.

2. In greater and greater proportions, the development of interpersonal skills and knowledges on the part of the student has become the responsibility of educational institutions. This is especially true for higher education because of the specialized categories for which they purport to train students. From the point of view of social functioning, institutions of higher education serve the basic function of providing training to those individuals who aspire to a higher status standing; they are societal instruments to facilitate upward social mobility.

3. Colleges and Universities, by and large, are failing to develop the essential interpersonal skills and knowledges on the part of the student which are essential for occupancy of the higher social category to which they aspire. We have no wellformulated, systematic training program to guarantee the student an opportunity to learn those interpersonal skills and knowledges essential and necessary for successful occupancy of the status for which we train him by way of personal skills and knowledges. This failure has, in part, been due to the myth that we in America have a classless society.

4. Historically, the student personnel worker has assumed major responsibility for those organizations which the college community has evolved to provide some training in the development of interpersonal skills. In any evaluation of current college personnel work the chief negative criticism which can be made is to the effect that, by and large, personnel workers have not developed more effective training procedures for imparting to the student the interpersonal skills and knowledges essential for occupancy of the status for which the academic faculty prepares him by way of personal skills and knowledges.

5. This failure of our educational institutions to develop 
more efficient ways of imparting the essential interpersonal skills and knowledges, or to at least explicitly bring them out in the open for the student to discuss, has resulted in an actual perpetuation of many arbitrary and undesirable, but, nevertheless, real social class differences. Our failure has resulted in a greater social and cultural lag which tends to interfere with the practices of truly democratic principles. Equally important is the fact that the personal conflicts which the college graduate experiences as a result of this lack of training causes the class, "college graduates," to exhibit a greater number of symptoms of maladjustment than any other educational class in our population.

\section{Some Recent Research on Interpersonal Relations and its Implication for College Personnel Work}

One large and important area of research on human behavior is that one concerned with isolating the variables which determine the development of personal or non-social skills. Adequate reviews of the voluminous literature concerned with these problems are made yearly and there is no point to my "rehashing" them at this time. Suffice to say that we can do a real workmanlike job with the problems of training in this area. We can measure an individual's non-social skills and knowledges and we can make a fair estimate of his aptitude to learn those individual skills. This is possible as a result of the rather elaborate development of basic knowledge in the field of tests and measurements which has in turn permitted the development of corresponding personnel practices represented in the college testing services. This ability to measure basic factors related to non-social skills has also permitted the development of elaborate job descriptions which define the minimal basic personal skills and abilities which are prerequisite for success on the job. This, in turn, has made possible a corresponding development of refined job-placement procedures by our college placement bureaus. We have a considerable body of basic knowledge concerning the variables which influence the acquisition of those personal skills which permit us to develop the corresponding college personnel practice of providing remedial training programs in the fields of reading, speech, English and mathematics, to name a few.

The body of basic scientific knowledge concerning the vari- 
ables which control the development of interpersonal skills and knowledge has, by contrast with the non-social skills, been slow in developing. However, in recent years significant studies have been made which have not, as yet, received adequate attention in educational circles. These studies do not provide the solution for effective training in interpersonal skills and knowledges. They do, however, suggest the kinds of further research which will eventually permit us to develop interpersonal skills as effectively as we can now develop personal skills. I would like to consider briefly some of these studies and to point out some of the implications they have for college personnel work.

The first field to be noted is that concerned with the development of measuring devices for assessing the interpersonal skills which an individual possesses. The most important development in this area has been the projective techniques. The projective technique on which we have the most research data is the Rorschach Test, which has served as the prototype for the other tests which have been developed in the projective field. Perhaps the best discussion of the projective techniques is to be found in a recent book by Bell (I). In addition to the wide use which has been made of projective tests in the personal counseling bureaus of colleges and universities, there have been some applications to the more general problems of college personnel work: for example, the work of Murphy at Sarah Lawrence (19); the work at the University of Chicago Medical School (I8); my own work in the medical school at the University of Oklahoma (26). The chief difficulty at present encountered with the projective tests is that we do not quite know what to do with the results. To demonstrate that the current procedures used to select freshman classes for Schools of Medicine result in a selection of students, of which approximately 20 per cent are definitely maladjusted, is one thing. To provide effective preventive training procedures in the pre-medical training program is quite another. There is, however, some basic research work which points the way in which these preventive and remedial training programs might develop, which brings us to the second research field which is concerned with attempts to identify the social variables which control the development of interpersonal skills.

Of major importance is the area of research represented by 
the classic studies of Sherif $(23,24)$. Sherif experimentally demonstrated that the activities of an individual are always a reflection of, and are influenced by, the social situation in which they occur. Secondly, his studies identify some of the basic variables which control the development of interpersonal ways of acting, and he has experimentally demonstrated the way in which some of these skills can be taught. These studies, since they experimentally identify some of the variables which control the development of interpersonal skills, should receive the considered attention of every personnel worker.

A second area is that represented by the work of Warner and his associates (28) which present data demonstrating conclusively that in our modern American communities there exist distinctive class and status structures, that each class and status has distinctive standards of acceptable behaviors, and that in moving from one class to another these different standards of behavior must result in much personal conflict. Perhaps an even more important contribution of Warner's Yankee City Series is the demonstration that ethnographic field methods can be successfully used, in complex societies, to isolate and describe those important cultural variables which determine the development of role behaviors acceptable to a given status. The implication for higher education of this work is clear. As social institutions designed to provide the student with the necessary skills to successfully engage in upward class mobility, it is essential that we know, first, what the role behaviors are that the student must learn, and second, how we can devise methods of teaching them. The Tankee City Series is a big step forward towards making both of these problems capable of solution.

A third area is that represented by the pioneering work of a small group of anthropologists, psychiatrists and psychologists on the relationship between personality development and social structure. Of importance here is the work of Ralph Linton (I6), H. S. Sullivan (27), Kardiner (II), Fromm (7), Gillin (8), Kaldegg (10), and DuBois (4). This work demonstrates conclusively that the individual's personality structure reflects the specific culture to which he has been exposed and that personality configurations vary as a function of the existing social structure. This has at least one important implication for edu- 
cational and personnel practices. We have in the United States cultures, not $a$ culture. It follows, therefore, that different colleges and universities, in order to achieve a common product must engage in varying educational procedures dictated by the cultural backgrounds of their students. A good methodology for developing desirable interpersonal skills on the part of the students, say at the University of Maine, may be of no value, or indeed a handicap, in attempting to achieve the same outcome at the University of Oklahoma. Each institution must develop its own program of social training in terms of the cultural background of its students. This conclusion strikes directly against one of the most hallowed of all personnel procedures, that of imitating in toto the procedures of a high prestige personnel organization located in some distant place, and justifying the whole procedure not in terms of local needs but in terms of the excellence of the program at the distant institution. Procedural imitation, as such, has no justification in terms of what we now know concerning regional cultural differences.

A fourth important area is that represented by the Bennington College study (20). This study demonstrated that attitudes, opinions, and ways of interpersonal participation can be modified by the interpersonal environment found in the College and that in large part this modification occurs in interpersonal activities engaged in external to the class room. The implication of this study for college personnel work is clear. The college personnel worker in his sphere of activities does have the means for developing successful training programs in interpersonal relations. His problem is simply one of research to isolate the variables which are dominant in his own particular institution. In this connection it should be noted that all college personnel research organizations have available the makings of an elaborate personnel research organization at their own institution, namely, the members of their own academic teaching staff. In line with an earlier discussion at this meeting, one of the easiest ways in which to obtain coöperation from the teaching staff at your institution is to provide them with a clear-cut research problem in their own field of research interest and ask their aid in its solution. Frankly, we of the academic staff are "suckers" for that kind of "bait" and, seriously, much valuable research data and goodwill would result from such coöperation. 
These major research trends for studying the variables controlling the development of interpersonal skills are actually concerned with the positive training of students in order to bring about an adequate mastery of essential interpersonal skills. There is one important aspect of training in interpersonal skills and knowledges left to discuss, namely, the field of remedial training.

The development of remedial procedures for training a student in interpersonal skills and knowledges is closely associated with the work of Freud. One of Freud's really great contributions to the treatment of the mentally ill was his recognition of the fact that most personal maladjustments develop from the conflicts arising in interpersonal relations, particularly the patterns of interpersonal relationships learned in the basic sociocultural group, the nuclear family. It was a comparatively easy step to adapt Freudian retraining procedures to the problem of the remedial training of interpersonal skills of college students. The emotional zeal and fervor with which the adaptations have been made by some college personnel people is evidence of the really great need those workers felt for some technique which would aid them with their retraining problems. One of the current popular but restricted and specialized adaptations of psychoanalytic procedure is called "non-directive counseling." Rogers (2r) in I $94^{2}$ published a manual summarizing a set of procedures which are derivations of some aspects of psychoanalytic techniques. In that manual he very carefully pointed out the limitations to the use of the technique in remedial training procedures. These limitations are such that the method could be strictly applied to only about io per cent of the general population and possibly I 5 per cent of the college population. Despite Roger's explicitly stated limitations there has occurred an almost hysterical generalization of those procedures to the point where some enthusiastic apostles of the non-directive technique offer this admittedly limited technique as a cure of all the ills of society. Even more unfortunate is the fact that hardly any basic research has been done to determine what the application of the technique actually does to the individual, or what validity the technique has for retraining the person in interpersonal skills.

What little evaluative research work has been done points to 
the restricting limitations which the method has. Take, for example, the generally held belief of the Rogerian apostles that: If given an unstructured situation in which one person talks to a second person concerning a personally frustrating problem there will result, on the part of the talker, an experiencing of emotional release or catharsis which, in turn, "provides for growth." "Growth" is the desired outcome. Translating the clique jargon into traditional psychological terms, it is held that verbalization about a frustrating problem will automatically result in the elimination of conflicting emotional factors which will in turn provide for improved performance in interpersonal situations. Yet, a recent experimental study (22) demonstrated conclusively that talk, in a Rogerian unstructured situation, about a personal problem served only to frustrate the individual more, which was reflected in a building up of an emotional state and a consequent lowering of the performance of the individual. Moreover, this study demonstrated that "emotional release" can occur only when the client ceases to be frustrated. The cessation of frustration may be due to problem solution or it may be brought about when the client temporarily escapes from talking about the frustrating problem: catharsis may occur without any problem solution. If nothing else, this study demonstrates the great urgent need for objective critical experimentation on the whole problem of the therapeutic role of emotional release and of the variables which govern its occurrence.

Much more clarification is also needed on what is meant by the stated desired end-product of counseling, "growth." Rogers and his students write about it as if growth takes place through the process of "insight." But insight is not a process, it is a phenomenon; no one has ever demonstrated a "process of insight." By inference "growth" must refer to psychological growth or learning, yet Rogers' manual contains not one single reference to the experimental studies on learning which have been accumulating for 55 years. Since therapy is synonymous with learning, it would seem reasonable that a consideration of the experimentally demonstrated facts concerning learning would aid greatly in the formulation of a methodology of retraining.

The whole problem of the validity of the claimed results of 
non-directive counseling is also in need of more careful experimental study. In a follow-up study I made of 50 employees at the Hawthorne plant, all of whom had been in counseling relationships with the counseling department for a year or more, I found 33 of them did show a permanent desirable change in their behavior as evidenced by a decrease in the number of personal problem references, etc. I also found that 26 of those 33 exhibited that change only after they had visited the Placement or Social Service Departments (which at Hawthorne have "directive counseling" functions). These departments brought about a re-structuring of the 26 employees' home or working interpersonal environment. In view of such data one can hardly claim that the desired change of all 33 was attributable solely to non-directive counseling.

In a recent monograph, Muench (17) purports to make an evaluation of non-directive therapy. The evaluation is made in terms of various test scores obtained before and after therapy. The size of the sample reported on is 12 . Certain statistical procedures were applied to the test results which are interpreted as showing that a desirable change occurred in the basic personality structures of this group which is attributable to non-directive psychotherapy. Of the tests used by Muench greatest emphasis is placed, perhaps rightly, on the Rorschach results. In grouping his data for analysis he assumed that during the process of therapy (i.e., the process of learning), evidences of "no-change" were insignificant and hence should not be considered in the statistical analysis. For example, he statistically evaluated the differences between the number of new Rorschach responses following therapy which were deemed desirable and the number of new Rorschach responses which were deemed undesirable following therapy. His conclusions were based on this type of analysis. This is analogous to assuming that in teaching the multiplication tables one has a successful method if, following training, the number of new correct answers given by students are greater than the number of additional new wrong answers given. The fact that a majority of the students continue to give the same answers at the end of training as they did at the beginning would, by Muench's reasoning, be ruled out as of no significance. If one makes the assumption, 
common to experimental studies of learning, that a failure to show a changed pattern of behavior or to show a change in the opposite direction from the onedesired is evidence of the failure of the training procedure, then one is justified in statistically comparing the improvement data with the "remainder" data. When such a grouping is made of Muench's Rorschach data (17, p. I 54) there results two groups, an "improvement" and a "no change plus decrement." Statistical analysis of those results shows that the Rorschach changes following therapy are just about what would be expected to occur due to chance, although in the "Personal Adjustment" factor there is a suggestion that personal adjustment was worse after counseling (difference statistically significant at the 20 per cent level of confidence). The number of cases involved in the Muench study was so small that one cannot attach too much significance to his results. His general design was laudable. With less biased treatment of the data it is possible that this design will provide much needed validity data concerning the technique.

Finally, there is a little-read, but important, monograph by Mary Shirley (25) which represents one of the most exacting validity studies of release therapy which has yet been reported. It reports a follow-up study which was made of 55 former clients of the Smith College Child Guidance Bureau. The follow-up was made from four to ten years following the closing of the clinic case record. Counseling had been given both children and parents. To quote the results found in the follow-up, "more than one-half of those children and mothers who received the type of guidance called 'advice and suggestion' eventually overcame their difficulties. ... only a tenth of the children and mothers who received 'insight' therapy at the clinic finally outgrew their difficulties."

I have gone in to the detailed consideration of the problem of the relative validity of "non-directive" counseling as a technique for developing interpersonal skills because I feel that not enough attention has been given to the whole important problem. Too much of the type of thinking represented by the "either-or" dichotomy has gone into the writings of its promoters. It is time that those personal counselors re-structured their field and came to appreciate that release therapy is just 
one of the many useful tools available for developing skills in interpersonal relations. A recent series of articles by Finesinger (5) attempts to demonstrate the way in which release therapy fits into the whole problem of remedial training of personality factors. "Non-directive" counseling, as one specific demonstration of a technique of release therapy, may demonstrate itself to be as useful a tool for developing certain deficient skills in habits of interpersonal relation as has Grace Fernald's kinesthetic reading method in the remedial training of certain deficient reading skills. One fact concerning its value is abundantly clear. At best, it is one of a host of useful tools available for remedial training of interpersonal skills; it is not the tool.

Finally, I wish to point out some methodological research procedures which are little used in college personnel work but which are worthy of further consideration by college personnel workers.

It is impossible to discuss methodology without immediately coming face to face with the problem of valid criteria. The validity of a given criterion is directly given by the accuracy with which one can predict a future specific event, (i.e., "event 2"), from a present specific event, (i.e., "event I"). A point which greatly simplifies the problem of valid criteria is that the empirical occurrence of event 2 is the only wholly valid criterion measure which exists. To the extent that we use as a criterion some measure of event 2 , to that extent we have a valid criterion. To the extent that we use some characteristic of event I which stands in a known empirical relationship to event 2, to that extent we are using valid criteria. These facts concerning the validity of criteria have important methodological implications. First, in order to make a statement concerning validity, one must be able to identify the two specific events under question. Second, these events must be quantifiable on a cardinal or ordinal scale. Third, we must know the empirical relationship which exists between events $I$ and 2 . 'To the extent that we attempt to make statements of relationship between concepts of high-level abstractions and specific events, to that extent we lower the validity of our stated relationship. This follows from the fact that the greater the degree of abstraction of the concept, the greater the probable error of the statement of relationship be- 
tween the concept itself and the specific event for which it stands. Thus, statements concerning "the relationship between childhood frustrations and success in life" are meaningless and the statement of relationship can have no validity since the abstraction "success in life" can mean almost any subsequent event. To discuss further the concept of validity would carry me far afield from my assigned topic. I hope that this brief discussion of the concept of validity will point to the importance of avoiding high-level abstractions and also point to the necessity for picking empirical observables when choosing criterion measures.

The first methodological procedures to be mentioned are those of R. A. Fisher and his students (6). His methods have been elaborated from the problems involved in more precisely estimating the magnitude of error introduced by random sampling, into an experimental situation. The actual statistical procedures utilized are called analysis of variance and covariance. In connection with his statistical work Fisher and his students have given detailed consideration to the types of experimental designs to which these methods of statistical analysis are best fitted. His designs, which are ideally fitted to many problems of research in college personnel work, have not achieved the popular recognition which they deserve. Since Fisher's methods permit one to validly estimate the degree of interaction occurring between two or more measurable variables, they are especially adaptable to research work in which several measurable variables are interacting simultaneously. Lindquist has pioneered the use of these methods in the field of education and his text (I4) is perhaps one of the best introductory presentations of the application of these methods to the problems of education.

The second methodological procedure is that involved in the ex post facto experiment. This methodological procedure consists essentially of a refinement and elaboration of aspects of the genetic method which has been used in psychology with varying degrees of crudeness since the time of G. Stanley Hall. The elaboration of the procedure into a very useful research method is attributable to a group of sociologists led by Professor F. S. Chapin of Minnesota (3). Perhaps the best statement of this method is to be found in a small volume by E. Greenwood (9). 
The nature of the ex post facto experimental design can perhaps be made clear by contrasting it with the classic single-variable experimental design in which we very carefully control and regulate the independent variable, (i.e., the stimulus variables) acting on an individual, and then observe the influence of that variable on the occurrence of event two, the response. Conversely, in the ex post facto design we exert our control through selection of the stimulus variable after it has operated and thereby reconstruct what might have been a classical experimental situation. The advantages of such a research design for personnel workers is very great since they commonly must start with the existing uncontrolled social and non-social environment of the students (i.e., the "environmental" variables) and are then asked to make meaningful explanatory statements concerning the resulting behavior.

The third methodological research procedure consists of the ethnological field methods which have been devised by anthropologists for studying culture. Briefly, their research method consists of actual partial initiation of the researcher into the role behaviors required by the statuses existing in a social group. The initiation takes place through the aid of an informant who is thoroughly familiar with the group. In practice, it is as if a Dean of Students was to go to a campus where he was not known, enroll in the college and then obtain an outstanding senior to guide and explain to him the proper behaviors to achieve the status of "outstanding senior." This would include an explanation of why it is important to efficiently use the files of past examinations; why it is necessary to work furiously to get admitted to an office in a social group, and, when once achieved, why it is doubly important to furiously work for an office in another social group; an explanation of the intricacies of the rating-dating complex, etc. In addition to the instructions given by the informant, this information would be supplemented by the visiting dean's own observations and his less intensive interviewing of other members of the society. He would continue to gather information on the culture until the number of novel, unexplained situations encountered per week or month stabilized around zero. This point might be represented graphically by that point on a negative growth curve at which the 
number of novel answers is constant and hence the curve becomes asyntotic to the base line around zero.

The chief advantage of the anthropological field methods for studying our college culture lies in the fact that, if properly used, they permit one to obtain a factual description of what actually are the shared patterns of interrelationships operating in the social group. This empirical type of descriptive data is, and must always be, the starting point in formulating a meaningful statement of the relationships existing between variables.

The chief criticisms which have been leveled at the procedures of the field anthropologist are: (I) their sampling techniques are wholly inadequate in that they are biased and result in distorted pictures; (2) they fail to present their data in terms of the individual but instead report their data in terms of categories or statuses; and, (3) that the methods are valid for studying primitive cultures but are wholly inadequate for dealing with complex cultures such as ours. The first and second criticisms have been made by naive individuals who are unaware of the fact that there are several equally valid levels at which relationships can be described. One does not have to describe interpersonal relationships in terms of the individual; they may be described in terms of categories or statuses and hence indirectly may be related to individuals just as we, in psychology, describe the independent variables controlling the rate of acquisition of a motor skill without any direct reference to the muscle segments involved. The common-sense idea of the anthropologist in the field, to consider his data complete when, with continued information-gathering activity, his basic pattern of information is not altered, is a sound procedural practice as has been demonstrated in a far more precise manner by the statistical formula for determining the size of a sample within given limits of sampling error-a formula that has proven valuable in phases of market research (2), public opinion polling (I 5), and studying sex behavior of the human male (12). By and large it appears that the field methods of the anthropologist have much to commend them to the personnel worker as a technique for obtaining descriptive information about interpersonal relationships. It is more than an interview technique since independent observations are constantly being made by the ethnographer 
and added to his body of knowledge concerning the culture being dealt with. The successful use of the method demands a rigorous application of the basic rules of scientific description just as does any other scientific methodology. The first volume of the Yankee City Series (28) and Kluckhohn's (13) recent monograph are perhaps the best descriptive sources on this technique.

In closing, higher education in general and college personnel workers in particular are going to have to develop more effective training programs for the development of interpersonal skills. To do this we must have, first of all, a body of basic knowledge concerning the variables controlling the development of interpersonal skills and knowledges. The social structure of the college provides an excellent laboratory. The college personnel and academic teaching staff working together as a research team can do the research. By such efforts, we may some day be able to write a job description of interpersonal skills and knowledges which is as complete a guide for training as are our present job descriptions of non-social skills and knowledges.

\section{REFERENCES}

I. Bell, J. E. Projective Techniques. New York: Longmans Green, I 948 .

2. Brown, T. H. Use of Statistical Technique in Certain Problems of Market Research. Cambridge: Harvard Univ. Press, I935.

3. Chapin, F. S. "Design for Social Experiments." American Sociological Review, III (1938), 786-800.

4. Du Bois, Cora A. The People of Alor. Minneapolis: The Univ. of Minnesota Press, 1944.

5. Finesinger, J. E. "Psychiatric Interviewing. I. Some Principles and Procedures in Insight Therapy." American Fournal of Psychiatry, CV (1948), 187-195.

6. Fisher, R. A. The Design of Experiments. Edinburgh: Oliver and Boyd, 1935.

7. Fromm, E. Escape from Freedom. New York: Farrar and Rinehart, I94r.

8. Gillin, J. "Cultural Adjustment." American Anthropology, XLVI (I944), 429-447.

9. Greenwood, E. Experimental Sociology: A Study in Method. New York: Kings Crown Press, 1945.

10. Kaldegg, A. "Responses of German and English Secondary School Boys to a Projection Test." British Fournal of Psychology, XXXIX (1948), 30-53.

I1. Kardiner, A., et al. The Psychological:Frontiers of Society. New York: Columbia Univ. Press, 1945. 
12. Kinsey, A. C., et al. Sexual Behavior in the Human Male. Philadelphia: IV. B. Saunders Co., 1948.

13. Kluckhohn, C. "The Personal Document in Anthropological Science." Bulletin of the Social Science Research Council, No. 53,1945 .

14. Lindquist, E. F. Statistical Analysis in Educational Research. Boston: Houghton Mifflin Co., I940.

15. Link, H. C. "How Many Interviews are Necessary for Results of a Certain Accuracy?" Fournal of Applied Psychology, XXI (1937), I-I7.

16. Linton, R. The Cultural Background of Personality. New York: Appleton-Century Co., I945.

17. Muench, G. A. "An Evaluation of Non-directive Psychotherapy by Means of the Rorschach and Other Indices." Applied Psychology Monograph, No. 13, I947.

18. Mullin, F. J. "Selection of Medical Students." Fournal of the Association of American Medical Colleges, XXIII (1948), 163I 70 .

19. Murphy, Lois B. Emotional Factors in Learning. New York: Columbia Univ. Press, 1944.

20. Newcomb, T. M. Personality and Social Change. New York: Dryden Press, 1943.

21. Rogers, C. R. Counseling and Psychotherapy. New York: Houghton Mifflin, 1942.

22. Rohrer, J. H. and Rayhill, Carol A. "Some Relationships Between Reminiscence and Catharsis." American Psychologist, I (1946), 460 .

23. Sherif, M. The Psychology of Social Norms. New York: Harper Brothers, 1936.

24. Sherif, M. "An Experimental Approach to the Study of Attitudes." Sociometry, I ( 1937 ), 90-98.

25. Shirley, Mary, Baum, Betty, and Polsky, Sylvia. "Outgrowing Childhood's Problems: A Followup Study of Child Guidance Patients." Smith College Study in Social Work, XI (I940), $31-60$.

26. Shoemaker, H. A. and Rohrer, J. H. "Relationship Between Success in the Study of Medicine and Certain Psychological and Personal Data." Fournal of the Association of American Medical Colleges, XXIII (1949), 190-201.

27. Sullivan, H. S. Conceptions of Modern Psychiatry. Washington: William A. White Psychiatric Foundation, I947.

28. Warner, W. L. and Lunt, P. S. The Status System of a Modern Community. New Haven: Yale Univ. Press, 1942. 